\title{
Depression, Anxiety, and Suicidal Ideation in Chinese University Students During the COVID-19 Pandemic
}

\author{
Shuang-Jiang Zhou' ${ }^{1+}$, Lei-Lei Wang ${ }^{11}$, Meng Qi ${ }^{2}$, Xing-Jie Yang', Lan Gao', \\ Suo-Yuan Zhang ${ }^{1}$, Li-Gang Zhang ${ }^{1}$, Rui Yang ${ }^{3}$ and Jing-Xu Chen ${ }^{1 *}$
}

${ }^{1}$ Beijing HuiLongGuan Hospital, Peking University HuiLongGuan Clinical Medical School, Beijing, China, ${ }^{2}$ Department of
Psychology, Chengde Medical University, Chengde, China, ${ }^{3}$ Beijing Anding Hospital, Capital Medical University, Beijing,
China

Coronavirus disease-2019 (COVID-19) pandemic has seriously threatened the global public health security and caused a series of mental health problem. Current research focuses mainly on mental health status and related factors in the COVID-19 pandemic among Chinese university students. Data from 11133 participants was obtained through an online survey. The Patient Health Question-9 (PHQ-9) was used to assess depressive symptoms, the Social Support Rate Scale (SSRS) was used to assess social support. We also used 7-item Generalized Anxiety Disorder Scale (GAD-7) to assess anxiety symptoms. Totally, 37.0\% of the subjects were experiencing depressive symptoms, 24.9\% anxiety symptoms, 20.9\% comorbid depressive and anxiety symptoms, and 7.3\% suicidal ideation. Multivariable logistic regression analysis revealed an increased presence of mental health problems in female students, graduate students, and those with personal COVID-19 exposure. Awareness of COVID-19, living with family were protective factors that reduced anxiety and depression symptoms. In addition, male, personal COVID-19 exposure, depressive and anxiety symptoms were risk factors for suicidal ideation. Social support, COVID-19 preventive and control measures, prediction of COVID-19 trends, living with family and graduate students are protective factors for reducing suicidal ideation.

Keywords: COVID-19, depressive symptoms, anxiety symptoms, suicidal ideation, social support

\section{INTRODUCTION}

Coronavirus disease-2019 (COVID-19) is an acute respiratory infection disease caused by severe respiratory syndrome coronavirus 2 (SARS-CoV-2). It is characterized by developing rapidly, widespread, and strong infectivity, and lack of specific treatment (Chan et al., 2020). The global COVID-19 epidemic is now nearly 1 year, with the coming of autumn and winter, COVID-19 is now worsening again in many countries. The COVID-19 epidemic has also caused many mental health problems (Bao et al., 2020). Since the COVID-19 outbreak, studies have shown that a high percentage of children, adolescents, and adults have psychological problems, such as suicidal tendencies, sleep disruption, anxiety, depression, and behavioral problems (Altena et al., 2020; Li et al., 2020; Liu et al., 2020; Purtle, 2020; Yang et al., 2020; Zhou et al., 2020). Some experts especially 
highlighted the urgency and importance of evaluating and managing mental health problems during the COVID-19 pandemic (Chenneville and Schwartz-Mette, 2020; Lai et al., 2020; Wang Y. et al., 2020). Social support is a resource in social relationships that may buffer or mitigate the effects of life events and other stressors (Kessler et al., 1985). Studies have shown that social support is associated with suicidal thoughts, anxiety, and depressive symptoms (Amit et al., 2020). High levels of social support can reduce suicidal ideation (Bi et al., 2020).

University students undergo a critical transition as they become independent and responsible for their own health during university years (Kim et al., 2018), and experience higher psychological stress levels (e.g., academic pressure, living conditions, financial situation) than their peers in the general population (Auerbach et al., 2018; Recabarren et al., 2019). University life is the peak period for the first onset of common mental disorders such as anxiety, depression and insomnia (Auerbach et al., 2018). This has brought a lot of troubles to university students, seriously affecting their social functions, study and life (Beiter et al., 2015; Scanlan and Hazelton, 2019; Jenkins et al., 2020). In addition, suicidal ideation are also common among university students (Lew et al., 2020). The anxiety and depression symptoms in college students are related to stress factors such as earthquakes, floods and epidemics (Huang et al., 2003; Li et al., 2003, 2011). University students' mental health problems have increased significantly during the outbreak of infectious diseases such as influenza A (H1N1) (Kanadiya and Sallar, 2011; Main et al., 2011).

During the outbreak of COVID-19, university students' education, including university studies and internship, was completed halted, which implies long hours at home and can lead to disordered rhythms of life and irregular sleep patterns. Moreover, the pandemic has brought the risk of infection and death. These may be traumatic experiences and have a psychological impact on this population. There have been no studies on anxiety, depressive symptoms, suicidal thoughts and social support among college students in China.

Since Chinese university students have been exposed to a persistent source of distress during the public health emergency, it is imperative to evaluate and respond to their mental health issues. But there have been no studies on anxiety, depressive symptoms, suicidal thoughts and social support among college students in China. For this purpose, the prevalence and potential factors contributing to depressive and anxiety symptoms, suicidal ideation, social supporting were detected.

\section{MATERIALS AND METHODS}

\section{Participants}

This research was a cross-sectional study, students were invited to complete a battery of online questionnaires through the Wenjuanxing platform from March 1 to 15, 2020. Inclusion criteria were full-time university students, including undergraduate and graduate students, living in mainland China, equal to or greater than 18 years of age. Participants who failed to complete the questionnaire were excluded from the study.
Students signed online informed consent before participating in the study. The study was also approved by the Ethics Committee of Beijing Huilongguan Hospital.

\section{Procedure \\ Sociodemographic Factors}

Demographic information, including gender, region, grade, and whether living with family were collected.

\section{Assessment of COVID-19 Exposure and Awareness of COVID-19}

Individual COVID-19 exposure was defined as a person who has been diagnosed with COVID-19, or a person who has a history of close contact with a COVID-19 patient in a mandatory isolation or medical observation. We used a self-made questionnaire to investigate university students' awareness of COVID-19. The questionnaire consisted of three main questions. The first question is about whether the subject is familiar with COVID-19. We asked the subject whether he/she has taken preventive and control measures to prevent COVID-19 infection for the second question. The final question asked the subject about his/her attitude toward the prediction of COVID-19 trends. The score for all the questions were ranged from 1 to 5 .

\section{Assessment of Depressive Symptoms}

We used the Chinese version of the 9-item Patient Health Questionnaire (PHQ-9) to assess the severity depressive symptoms (Spitzer et al., 1999). The questionnaire consists of 9 items, For each item, the answer consists of four choices: Not at all, several days, more than a week, and almost every day. The corresponding score is $0,1,2$, and 3 . The symptom severity is determined by the total score, with 5-9 being mild, 10-14 being moderate, 15-19 being moderately severe, and 20-27 being severe.

\section{Assessment of Anxiety Symptoms}

We used Chinese version of the 7-item Generalized Anxiety Scale (GAD-7) to assess participants' anxiety symptoms (Spitzer et al., 2006), with symptom prevalence on a scale from 0 (not at all) to 3 (nearly every day). The symptom severity is determined by the total score, with 5-9 being mild, 10-14 being moderate, and 15-21 being severe.

\section{Assessment of Suicidal Ideation}

Suicidal ideation among college students was assessed by single item (item 9) of PHQ-9, which asked participants how often they thought they would be better off dead. Suicidal ideation is divided into four grades: From 0 (not at all) to 3 (nearly every day). The higher the level, the more serious the suicidal ideation.

\section{Assessment of Social Support}

The social support scale developed by Xiao Shuiyuan was used to evaluate the social support of college students. There are 10 items in this scale, including subjective support, objective support and utilization of support. A higher score indicates a higher level of social support or utilization. Previous studies have shown that the social support scale has good reliability and validity (Shuiyuan, 1987). 


\section{Data Analysis}

We used SPSS 24.0 for data analysis. We use percentages to show the proportion of depression symptoms, anxiety symptoms, suicidal ideation. The chi-square test was used to analyze the categorical variables. Logistic regression was used to explore the predictors of depressive or anxiety symptoms, and suicidal ideation. The level of significance was set at $p<0.05$ (two-sided).

We used the Process macro program in SPSS to conduct the mediation effect analysis. In the mediating effect model, whether there is COVID-19 exposure is an independent variable, suicide concept is a dependent variable, subjective support and objective support are mediators. The results of the mediation analysis are presented in the form of plots. We used bootstrap to test the mediating effect. The sample size was set to 5,000, and the $95 \%$ confidence interval of indirect effect did not include zero, indicating that the mediating effect was significant.

\section{RESULTS}

A total of 11,372 participants completed the online questionnaires. After removing those answering less than 3 min or living abroad, 11,133 participants (18-35 years old, median = 21) from 31 provincial-level regions of mainland China, except Macau and Hong Kong, were involved in the current study, giving a response prevalence of $97.9 \%$. Table 1 shows that $62.3 \%$ of the participants were female, $56.4 \%$ were urban residents, $90.3 \%$ were graduate students, $95.5 \%$ were living with their families, and 7.2\% had exposure to COVID-19.

A total of $37.0 \%$ participants experienced mild to severe depressive symptoms, $24.9 \%$ experienced mild to severe anxiety symptoms, and the comorbidity prevalence of depressive and anxiety symptoms was $20.9 \%$. Moreover, $7.3 \%$ of the students had suicidal ideation. The distribution of age among the three groups: With and without symptoms of depression, with and without symptoms of anxiety, with and without suicidal thoughts was non-normal ( $P<0.001$ for all Kolmogorov-Smirnova tests), so the Non-parametric Mann-Whitney test was used to compare the ages of all three groups. But only the group with anxiety symptoms was older than those without anxiety symptoms group $(P<0.001)$. As shown in Table 1 , there were no differences in depressive and anxiety symptoms, suicidal ideation among university students between different regions. The proportion of depressive symptoms and anxiety symptoms among female students was higher than male students (38.9 vs. $33.9 \%$; 26.1 vs. $22.8 \%)$. But the proportion of suicidal ideation for male students was higher than female students ( 8.1 vs. 6.8\%). Depressive and anxiety symptoms were more likely to occur in graduate students than in undergraduates ( 41.2 vs. $36.5 \% ; 29.6$ vs. $24.3 \%$ ), but there was no difference between undergraduate and graduate students for suicidal ideation. The differences in depressive and anxiety symptoms, suicidal ideation between students living with and without their families were statistically significant (53.3 vs. $36.2 \%$; 42.6 vs. $24.0 \%$; 19.6 vs. $6.7 \%$ ). Students with COVID19 exposure reported more depressive and anxiety symptoms, suicidal ideation than those without COVID-19 exposure (46.1 vs. $36.3 \%$; 34.0 vs. $24.1 \%$; 10.5 vs. $7.0 \%)$.
As shown in Table 2, The higher scores of COVID-19 awareness, preventive and control measures, and COVID19 trend prediction scores, the lower proportion of anxiety symptoms, depression symptoms, and suicidal ideation.

Table 3 multivariable logistic regression showed that there was an increased presence of depressive and anxiety symptoms in female students $\left(\mathrm{OR}_{\mathrm{D}}=1.24,95 \% \mathrm{CI}: 1.14-1.34 ; \mathrm{OR}_{\mathrm{A}}=1.21\right.$, 95\% CI: $1.11-1.33)$, graduate students $\left(\mathrm{OR}_{\mathrm{D}}=1.14,95 \% \mathrm{CI}: 1.00-\right.$ $\left.1.30 ; \mathrm{OR}_{\mathrm{A}}=1.18,95 \% \mathrm{CI}: 1.02-1.346\right)$, and those with COVID-19 exposure $\left(\mathrm{OR}_{\mathrm{D}}=1.42,95 \% \mathrm{CI}: 1.22-1.65 ; \mathrm{OR}_{\mathrm{A}}=1.51,95 \%\right.$ CI: 1.29-1.76). We found that college students living with their parents $\left(\mathrm{OR}_{\mathrm{D}}=0.52,95 \% \mathrm{CI}: 0.44-0.63 ; \mathrm{OR}_{\mathrm{A}}=0.46,95 \% \mathrm{CI}\right.$ : $0.38-0.55)$, being familiar with COVID-19 $\left(\mathrm{OR}_{\mathrm{D}}=0.85,95 \%\right.$ CI: $\left.0.81-0.89 ; \mathrm{OR}_{\mathrm{A}}=0.92,95 \% \mathrm{CI}: 0.87-0.97\right)$, actively taking preventive and control measures $\left(\mathrm{OR}_{\mathrm{D}}=0.88,95 \% \mathrm{CI}: 0.84-\right.$ $\left.0.92 ; \mathrm{OR}_{\mathrm{A}}=0.87,95 \% \mathrm{CI}: 0.83-0.91\right)$, and being optimistic about projections of COVID-19 trends $\left(\mathrm{OR}_{\mathrm{D}}=0.71,95 \% \mathrm{CI}\right.$ : $\left.0.67-0.74 ; \mathrm{OR}_{\mathrm{A}}=0.67,95 \% \mathrm{CI}: 0.63-0.70\right)$ were protective factors for depressive symptoms and anxiety symptoms. As for suicidal ideation, multivariable logistic regression showed that depressive $(\mathrm{OR}=10.62,95 \% \mathrm{CI}: 7.84-14.38)$ and anxiety symptoms ( $\mathrm{OR}=5.56,95 \% \mathrm{CI}$ : 4.53-6.81) were risk factors. And female students $(\mathrm{OR}=0.72,95 \% \mathrm{CI}$ : 0.61-0.84), graduate students $(\mathrm{OR}=0.74,95 \% \mathrm{CI}$ : $0.57-0.97)$, living with family (OR $=0.48,95 \%$ CI: 0.37-0.64), preventive and control measures $(\mathrm{OR}=0.86,95 \% \mathrm{CI}: 0.75-0.91)$, and projections of COVID-19 trends $(\mathrm{OR}=0.83,95 \% \mathrm{CI}: 0.60-0.71)$.

Linear regression showed that COVID-19 exposure was negatively correlated with subjective support, objective support, and suicidal thoughts. There was also a negative correlation between subjective support, objective support, and suicidal thoughts. Based on the regression results, we established a mediating effect model. As shown in Figures 1, 2 (models 1 and 2), the indirect effects between COVID-19 exposure and suicidal ideation through objective support, subjective support were significant, suggesting that models 1 and 2 were full mediation models.

\section{DISCUSSION}

Emotional problems are the most common psychological symptoms in university students (Auerbach et al., 2016), which may further increase during public health emergencies (Cao et al., 2020). This survey indicated the following main findings. Firstly, among university students in mainland China during the COVID-19 pandemic, 37.0\% experienced depressive symptoms, $24.9 \%$ experienced anxiety symptoms, and $20.9 \%$ experienced comorbidity depressive and anxiety symptoms. Secondly, female gender, being a graduate, and personal COVID-19 exposure were independent risk factors and living with family was an independent protective factor for developing depressive and anxiety symptoms. Thirdly, awareness of COVID-19 is an important factor in reducing anxiety and depression symptoms, and suicide ideation.

In general, the prevalence of depressive and anxiety symptoms demonstrated in this study is clearly much higher than that 
TABLE 1 | Socio-demographic characteristics and association with depressive and anxiety symptoms.

\begin{tabular}{|c|c|c|c|c|c|c|c|c|c|c|c|}
\hline \multirow[t]{2}{*}{ Variables } & \multirow[t]{2}{*}{$\mathbf{n}$} & \multirow[t]{2}{*}{$\%$} & \multicolumn{3}{|c|}{ Depressive symptoms } & \multicolumn{3}{|c|}{ Anxiety symptoms } & \multicolumn{3}{|c|}{ Suicidal ideation } \\
\hline & & & $\mathbf{N}$ & $\%$ & $P$ & $\mathbf{n}$ & $\%$ & $P$ & $\mathbf{n}$ & $\%$ & $p$ \\
\hline Gender & & & & & $<0.001$ & & & $<0.001$ & & & 0.007 \\
\hline Male & 4,195 & 37.7 & 1,424 & 33.9 & & 956 & 22.8 & & 341 & 8.1 & \\
\hline Female & 6,938 & 62.3 & 2,695 & 38.8 & & 1,811 & 26.1 & & 469 & 6.8 & \\
\hline Region & & & & & 0.165 & & & 0.866 & & & 0.080 \\
\hline Urban resident & 6,284 & 56.4 & 2,360 & 37.6 & & 1,558 & 24.8 & & 481 & 7.7 & \\
\hline Rural resident & 4,849 & 43.6 & 1,759 & 36.3 & & 1,209 & 24.9 & & 329 & 6.8 & \\
\hline Grade & & & & & 0.003 & & & $<0.001$ & & & 0.958 \\
\hline Undergraduates & 10,053 & 90.3 & 3,674 & 36.5 & & 2,477 & 24.3 & & 731 & 7.3 & \\
\hline Graduate students & 1,080 & 9.7 & 445 & 41.2 & & 320 & 29.6 & & 79 & 7.3 & \\
\hline Living with family & & & & & $<0.001$ & & & $<0.001$ & & & $<0.001$ \\
\hline Yes & 10,628 & 95.5 & 3,850 & 36.2 & & 2,552 & 24.0 & & 711 & 6.7 & \\
\hline No & 505 & 4.5 & 269 & 53.3 & & 215 & 42.6 & & 99 & 19.6 & \\
\hline COVID-19 exposure & & & & & $<0.001$ & & & $<0.001$ & & & $<0.001$ \\
\hline Yes & 801 & 7.2 & 369 & 46.1 & & 272 & 34.0 & & 84 & 10.5 & \\
\hline No & 10,332 & 82.8 & 3,750 & 36.3 & & 2,495 & 24.1 & & 726 & 7.0 & \\
\hline Total & 11,133 & 100 & 4,119 & 37.0 & & 2,767 & 24.9 & & 810 & 7.3 & \\
\hline
\end{tabular}

TABLE 2 | The relationship between COVID-19 awareness and depressive and anxiety symptoms.

\begin{tabular}{|c|c|c|c|c|c|c|c|c|c|c|c|}
\hline \multirow[t]{2}{*}{ Variables } & \multirow[t]{2}{*}{$\mathbf{n}$} & \multirow[t]{2}{*}{$\%$} & \multicolumn{3}{|c|}{ Depressive symptoms } & \multicolumn{3}{|c|}{ Anxiety symptoms } & \multicolumn{3}{|c|}{ Suicidal ideation } \\
\hline & & & $\mathbf{n}$ & $\%$ & $P$ & $\mathbf{n}$ & $\%$ & $P$ & $\mathbf{n}$ & $\%$ & $P$ \\
\hline COVID-19 knowledge & & & & & $<0.001$ & & & $<0.001$ & & & $<0.001$ \\
\hline Very unfamiliar & 128 & 1.1 & 66 & 51.6 & & 45 & 35.2 & & 23 & 18.0 & \\
\hline Unfamiliar & 1,606 & 14.4 & 674 & 42.0 & & 440 & 27.4 & & 132 & 8.2 & \\
\hline Medium level & 4,713 & 42.3 & 1,836 & 39.0 & & 1,203 & 25.5 & & 361 & 7.7 & \\
\hline Familiar & 3,777 & 33.9 & 1,290 & 34.2 & & 908 & 24.0 & & 226 & 6.0 & \\
\hline Very familiar & 909 & 8.2 & 253 & 27.8 & & 171 & 18.8 & & 68 & 7.5 & \\
\hline Preventive and control measures & & & & & $<0.001$ & & & $<0.001$ & & & $<0.001$ \\
\hline Very inconsistent & 202 & 1.8 & 68 & 33.7 & & 53 & 26.2 & & 22 & 10.9 & \\
\hline Inconsistent & 462 & 4.1 & 196 & 42.4 & & 462 & 27.1 & & 42 & 9.1 & \\
\hline Neutral & 899 & 8.1 & 409 & 45.5 & & 304 & 33.8 & & 103 & 11.5 & \\
\hline Consistent & 5,909 & 53.1 & 2,300 & 38.9 & & 2,536 & 26.0 & & 449 & 7.6 & \\
\hline Very consistent & 3,661 & 32.9 & 1,146 & 31.3 & & 749 & 20.5 & & 194 & 5.3 & \\
\hline Projections of COVID-19 trend & & & & & $<0.001$ & & & $<0.001$ & & & $<0.001$ \\
\hline Very pessimistic & 99 & 0.9 & 49 & 49.5 & & 42 & 42.4 & & 19 & 19.2 & \\
\hline Pessimistic & 734 & 6.6 & 397 & 54.1 & & 306 & 41.7 & & 101 & 13.8 & \\
\hline Neutral & 2,660 & 23.9 & 1,162 & 43.7 & & 823 & 30.9 & & 266 & 10.0 & \\
\hline Optimistic & 6,434 & 57.8 & 2,209 & 34.3 & & 1,410 & 21.9 & & 373 & 5.8 & \\
\hline Very optimistic & 1,206 & 10.8 & 302 & 25.0 & & 186 & 15.4 & & 51 & 4.2 & \\
\hline
\end{tabular}

in most previous studies during non-pandemic periods. For example, a meta-analysis, involving 39 studies with 32694 Chinese university students, indicated that the prevalence of depressive symptoms was $23.8 \%$ (95\% CI: 19.9-28.5\%) (Lei et al., 2016). Studies have shown that about $10 \%$ of undergraduate and graduate students report significant anxiety symptoms during their school years (Eisenberg et al., 2013; Auerbach et al., 2016). However, a relatively high prevalence of depressive and anxiety symptoms has also been observed in individual studies (Othman et al., 2019). On further analysis of the severity of mental health problems, it was found that mild depressive and anxiety symptoms were most common. In addition to anxiety and depression symptoms, college students' suicidal ideation during the COVID-19 epidemic should also be concerned. Studies have shown that during the COVID-19 epidemic, the public has a high rate of suicidal ideation due to factors such as unemployment, home isolation, anxiety, depression, and insomnia symptoms (Bryan et al., 2020; Kawohl and Nordt, 2020; Li et al., 2020). But there have been no studies of college students. So it is worth mentioning that, even though only $7.3 \%$ students had suicide ideation, more attention should be paid to students with these characteristics. 
TABLE 3 | Sociodemographic characteristics and COVID-19 awareness correlates with depressive and anxiety symptoms.

\begin{tabular}{|c|c|c|c|c|c|c|c|c|c|}
\hline \multirow[t]{2}{*}{ Variables } & \multicolumn{3}{|c|}{ Depressive symptoms } & \multicolumn{3}{|c|}{ Anxiety symptoms } & \multicolumn{3}{|c|}{ Suicidal ideation } \\
\hline & OR & $95 \% \mathrm{Cl}$ & $P$ & OR & $95 \% \mathrm{Cl}$ & $P$ & OR & $95 \% \mathrm{Cl}$ & $P$ \\
\hline \multicolumn{10}{|l|}{ Gender } \\
\hline Male & 1 & & & 1 & & & 1 & & \\
\hline Female & 1.24 & $1.14-1.34$ & $<0.001$ & 1.21 & $1.11-1.33$ & $<0.001$ & 0.72 & $0.61-0.84$ & $<0.001$ \\
\hline \multicolumn{10}{|l|}{ Grade } \\
\hline Undergraduate & 1 & & & 1 & & & 1 & & \\
\hline Graduate & 1.14 & $1.00-1.30$ & 0.051 & 1.18 & $1.02-1.36$ & 0.025 & 0.74 & $0.57-0.97$ & 0.028 \\
\hline \multicolumn{10}{|l|}{ Living with family } \\
\hline No & 1 & & & 1 & & & 1 & & \\
\hline Yes & 0.52 & $0.44-0.63$ & $<0.001$ & 0.46 & $0.38-0.55$ & $<0.001$ & 0.48 & $0.37-0.64$ & $<0.001$ \\
\hline \multicolumn{10}{|l|}{ COVID-19 exposure } \\
\hline No & 1 & & & 1 & & & 1 & & \\
\hline Yes & 1.42 & $1.22-1.65$ & $<0.001$ & 1.51 & $1.29-1.76$ & $<0.001$ & 1.17 & $0.89-1.52$ & 0.262 \\
\hline \multicolumn{10}{|l|}{ Awareness of COVID-19 } \\
\hline COVID-19 knowledge & 0.85 & $0.81-0.89$ & $<0.001$ & 0.92 & $0.87-0.97$ & 0.001 & 1.00 & $0.91-1.09$ & 0.937 \\
\hline Preventive and control measures & 0.88 & $0.84-0.92$ & $<0.001$ & 0.87 & $0.83-0.91$ & $<0.001$ & 0.86 & $0.75-0.91$ & 0.001 \\
\hline Projections of COVID-19 trend & 0.71 & $0.67-0.74$ & $<0.001$ & 0.67 & $0.63-0.70$ & $<0.001$ & 0.83 & $0.60-0.71$ & $<0.001$ \\
\hline \multicolumn{10}{|l|}{ Depressive symptoms } \\
\hline No & - & & & - & & & 1 & & \\
\hline Yes & - & & & - & & & 10.62 & $7.84-14.38$ & $<0.001$ \\
\hline \multicolumn{10}{|l|}{ Anxiety symptoms } \\
\hline No & - & & & - & & & 1 & & \\
\hline Yes & - & & & - & & & 5.56 & $4.53-6.81$ & $<0.001$ \\
\hline
\end{tabular}

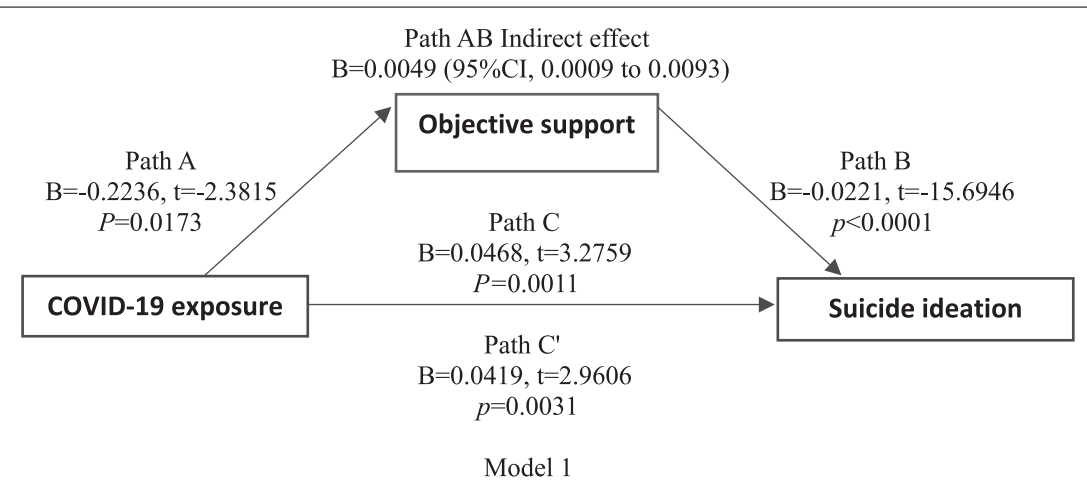

FIGURE 1 | Model 1 shoes path diagram of the mediation model (X=COVID-19 exposure; $Y=$ suicidal ideation). Path C represent the variance in COVID-19 exposure associated with suicidal ideation. Path C' represent the association between COVID-19 exposure and suicidal ideation after taking into account objective support. Path $\mathrm{AB}$ is the mediation effect and is significant at $P<0.05$.

There is now sufficient evidence to state that the female gender is a reliable risk factor for depressive and anxiety symptoms (Gater et al., 1998; Othman et al., 2019; Jenkins et al., 2020; Zhou et al., 2020). But our study found female students is a protect factor for suicidal ideation, which is consistent with previous research on factors influencing suicidal ideation among Chinese college students, it may be related to the great pressure placed on male college students by Chinese society (Lllhm, 2006). Graduate students, in contrast to undergraduate ones, have more negative emotions. This might be explained by more profound stresses regarding economic, marital, academic, interpersonal, and employment concerns as results of the pandemic. Although graduate students had more negative emotions, they had less suicidal ideation than undergraduates, this is not consistent with previous studies. Studies have shown that in the student population, for those older than 25 years old, the suicide rate of students is significantly higher than that of students younger than 25 years old. In the group of students aged 20-24, suicide rate of graduate students is higher than that of undergraduate students (Silverman et al., 1997; Hamilton and Schweitzer, 2000). In the present study, students living with family are related to lower risk of mental health problems, lower percentage of suicidal ideation. Some authors have demonstrated that family support, especially parental support, is very important and could effectively buffer 


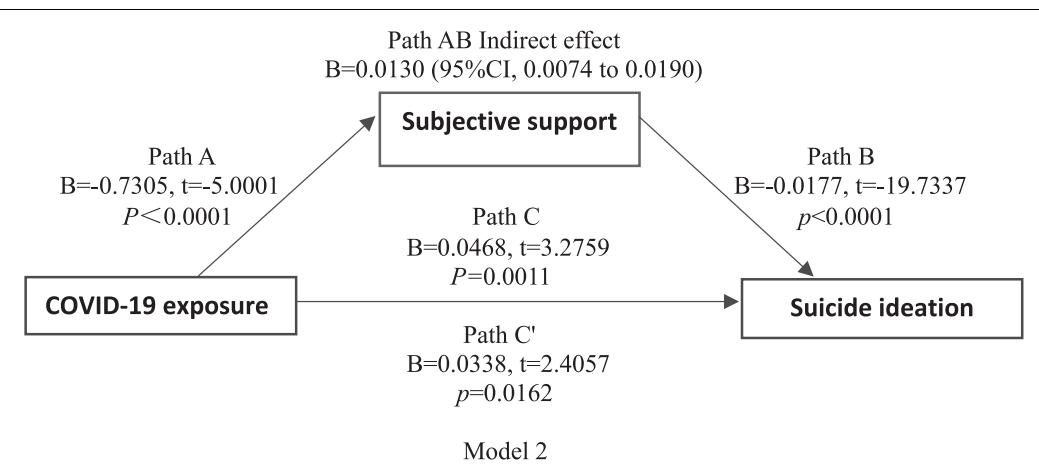

FIGURE 2 | Model 2 shoes path diagram of the mediation model (X=COVID-19 exposure; $Y=$ suicidal ideation). Path C represent the variance in COVID-19 exposure associated with suicidal ideation. Path C' represent the association between COVID-19 exposure and suicidal ideation after taking into account subjective support. Path $\mathrm{AB}$ is the mediation effect and is significant at $P<0.05$.

the effects of high stress on anxiety symptoms and depressive symptoms, it also reduces suicidal ideation (Crockett et al., 2007; Gariepy et al., 2016; van Harmelen et al., 2016; Pruitt et al., 2020). Conversely, emotional loneliness caused by family disconnection is an important factor leading to mental health problems (Fernandez-Rouco et al., 2019). As predicted, COVID19 exposure is closely related to bad moods. Individuals who were quarantined, irrespective of their wishes, suffered from isolation and directly faced the problems of infection, medical treatment, and even death (Elizarraras-Rivas et al., 2010; Oboho et al., 2015). But we also found that objective support, subjective support, was the intermediary between COVID-19 exposure and suicidal ideation. Previous studies have also shown that high levels of social support are protective factors for suicidal ideation (Hirsch and Barton, 2011; Parker et al., 2021). Therefore, providing social support to college students during the COVID-19 epidemic, especially for college students exposed to COVID-19, can reduce suicidal ideation.

Good awareness regarding infectious diseases may assist in the prevention of psychological problems (Khan et al., 2015). More accurate COVID-19 knowledge can reduce negative attitudes, potentially dangerous practices, fear and panic during the epidemic (Ren et al., 2020). Our findings supported this view and revealed COVID-19 awareness as an independent protective factor for mental health among university students. Of course, it is important to provide timely, specific and accurate health information about COVID-19 (Wang C. et al., 2020). Since the early stage of the COVID-19 pandemic, the Chinese government has provided essential COVID-19 knowledge to the public every day, through media campaigns via television, radio, WeChat, Tik Tok, and newspapers. However, it was found that only $42.1 \%$ students were familiar and $15.5 \%$ were unfamiliar with COVID19 knowledge. Therefore, public health policy makers and health workers should attach importance to COVID-19 prevention training and health education for university students.

Based on the pandemic characteristics of COVID-19, the Chinese government and public authorities made efforts to facilitate the implementation of pandemic prevention measures. The practices were very cautious in the Chinese population: Decreased unnecessary outings, avoiding crowded places, wearing masks when going outside, and washing hands frequently (Zhong et al., 2020). Our study results were in agreement with a previous study, which suggested that precautionary measures could reduce the levels of anxiety and depression symptoms and psychological impact of the outbreak (Leung et al., 2003; Wang C. et al., 2020; Xiang et al., 2020).

During this survey period, the number of reported infection cases nationwide began to decline slightly, but the pandemic was spreading rapidly around the world and some imported cases occurred. Therefore, the public was urged to take more stringent preventive and control measures. Almost all students continued to stop their university studies and practice, and their range of activities was greatly restricted, which caused great inconvenience in their lives. Long-term self-isolation can make people bored and prone to focus too much on negative pandemic information, which also increases the risk of mental health problems (Gostic et al., 2020). However, our finding that the majority of students had an optimistic attitude about overcoming this crisis was unexpected. The most likely explanations for this situation are the openness and transparency of data, the effective and standardized implementation of preventive and control work in China (China, 2020). The optimistic attitude toward the prospects of COVID-19 could reduce depressive and anxiety symptoms, since risk perception has a greater correlation with mental health (Liao et al., 2014). However, recently, there has been a rebound of COVID-19 epidemic abroad, and many schools are facing another shutdown and class closure. The worsening COVID-19 epidemic may cause psychological problems among college students again. Therefore, our research also has certain guiding significance to alleviate the psychological problems of college students.

The key strengths of this study included the wide-ranging demographics and the largest sample studied to date. In addition, it was the first study to investigate the prevalence of anxiety, depression symptoms and suicidal ideation among university students and its influence during the COVID-19 epidemic. However, there are also some limitations to this study. First, the study adopted the method of convenience sampling to recruit subjects, which may lead to a lack of sample representativeness and an imbalance of the sample distribution. Second, we used 
the self-assessment questionnaire to assess the symptoms of anxiety and depression, so that reporting bias may exist when compared with the professional assessment. Third, all the data were collected in a cross-sectional survey, and therefore, causal relationships could not be established. Finally, the item 9 of PHQ-9 was mainly used for the evaluation of suicidal ideation. No professional questionnaire is used for the evaluation of suicidal ideation, which may not be systematic and detailed enough.

\section{CONCLUSION}

In conclusion, the mental health status of university students has been affected during the COVID-19 pandemic, with a high prevalence of depressive symptoms, anxiety symptoms, and suicidal ideation. The female gender, graduates, living with family, personal COVID-19 exposure and awareness of COVID-19 were related factors for depressive and anxiety symptoms. In addition, our study showed that anxiety and depression symptoms are important risk factors for suicidal ideation. We also found that social support mediated between exposure and suicidal ideation. Providing adequate social support to university students may reduce suicide. While paying attention to the anxiety and depression symptoms of university students, we should also pay attention to the students' suicidal ideation, and focus on the intervention of students with suicidal ideation.

\section{DATA AVAILABILITY STATEMENT}

The data that supports the findings of this study are available from the corresponding author upon reasonable request.

\section{REFERENCES}

Altena, E., Baglioni, C., Espie, C. A., Ellis, J., Gavriloff, D., Holzinger, B., et al. (2020). Dealing with sleep problems during home confinement due to the COVID-19 outbreak: practical recommendations from a task force of the European CBT-I Academy. J. Sleep Res. 29:e13052. doi: 10.1111/jsr.13052

Amit, N., Ismail, R., Zumrah, A., Mohd Nizah, M., Tengku Muda, T., Tat Meng, E., et al. (2020). Relationship between debt and depression, anxiety, stress, or suicide ideation in asia: a systematic review. Front. Psychol. 11:1336. doi: 10.3389/fpsyg.2020.01336

Auerbach, R. P., Alonso, J., Axinn, W. G., Cuijpers, P., Ebert, D. D., Green, J. G., et al. (2016). Mental disorders among college students in the World Health Organization World Mental Health Surveys. Psychol. Med. 46, 2955-2970. doi: 10.1017/S0033291716001665

Auerbach, R. P., Mortier, P., Bruffaerts, R., Alonso, J., Benjet, C., Cuijpers, P., et al. (2018). WHO World Mental Health Surveys International College Student Project: prevalence and distribution of mental disorders. J. Abnorm. Psychol. 127, 623-638. doi: 10.1037/abn0000362

Bao, Y., Sun, Y., Meng, S., Shi, J., and Lu, L. (2020). 2019-nCoV epidemic: address mental health care to empower society. Lancet 395, e37-e38. doi: 10.1016/ S0140-6736(20)30309-3

Beiter, R., Nash, R., McCrady, M., Rhoades, D., Linscomb, M., Clarahan, M., et al. (2015). The prevalence and correlates of depression, anxiety, and stress in a

\section{ETHICS STATEMENT}

The studies involving human participants were reviewed and approved by the Ethics Committee of Beijing HuiLongGuan Hospital. The patients/participants provided their written informed consent to participate in this study.

\section{AUTHOR CONTRIBUTIONS}

S-JZ and X-JY completed the design of the questionnaire. MQ was responsible for the examination of the contents of the questionnaire. LG, S-YZ, and L-GZ were responsible for the distribution and recovery of the questionnaire. L-LW and J-XC completed the statistical analyses. $\mathrm{RY}$ and J-XC received funding support for the research. S-JZ and MQ jointly completed the first draft of this manuscript. J-XC designed the whole study, provided guidance and reviewed and submitted the article. All authors have read and agreed with the published version of the manuscript.

\section{FUNDING}

This work was supported by the Beijing Municipal Administration of Hospitals Clinical Medicine Development of Special Funding (XMLX201806), the Capital Foundation of Medicine Research and Development (2018-3-2132), and the Special Foundation of Beijing Municipal Science and Technology Commission (Z171100001017001).

\section{ACKNOWLEDGMENTS}

We thank all of subjects who participated in this study. We would like to thank Editage (www.editage.cn) for English language editing.

sample of college students. J. Affect. Disord. 173, 90-96. doi: 10.1016/j.jad.2014. 10.054

Bi, F., Luo, D., Huang, Y., Chen, X., Zhang, D., and Xiao, S. (2020). The relationship between social support and suicidal ideation among newly diagnosed people living with HIV: the mediating role of HIV-related stress. Psychol. Health Med. 26, 724-734. doi: 10.1080/13548506.2020.1761987

Bryan, C. J., Bryan, A. O., and Baker, J. C. (2020). Associations among state-level physical distancing measures and suicidal thoughts and behaviors among U.S. adults during the early COVID-19 pandemic. Suicide Life Threat. Behav. 50, 1223-1229. doi: 10.1111/sltb.12653

Cao, W., Fang, Z., Hou, G., Han, M., Xu, X., Dong, J., et al. (2020). The psychological impact of the COVID-19 epidemic on college students in China. Psychiatry Res. 287:112934. doi: 10.1016/j.psychres.2020.11 2934

Chan, J., Yuan, S., Kok, K., To, K., Chu, H., Yang, J., et al. (2020). A familial cluster of pneumonia associated with the 2019 novel coronavirus indicating person-to-person transmission: a study of a family cluster. Lancet 395, 514-523. doi: 10.1016/s0140-6736(20)30154-9

Chenneville, T., and Schwartz-Mette, R. (2020). Ethical considerations for psychologists in the time of COVID-19. Am. Psychol. 75, 644-654. doi: 10.1037/ amp0000661

China, N. H. C. (2020). Technical Guide for Prevention and Control of New Coronavirus Infection in Medical Institutions, 2nd Edn. Available online at: 
http://www.nhc.gov.cn/jkj/s3577/202001/c67cfe29ecf1470e8c7fc47d3b751e88. shtml (accessed January 22, 2020).

Crockett, L. J., Iturbide, M. I., Torres Stone, R. A., McGinley, M., Raffaelli, M., and Carlo, G. (2007). Acculturative stress, social support, and coping: relations to psychological adjustment among Mexican American college students. Cultur. Divers. Ethnic. Minor. Psychol. 13, 347-355. doi: 10.1037/1099-9809.13.4.347

Eisenberg, D., Hunt, J., and Speer, N. (2013). Mental health in American colleges and universities: variation across student subgroups and across campuses. J. Nerv. Ment. Dis. 201, 60-67. doi: 10.1097/NMD.0b013e31827ab077

Elizarraras-Rivas, J., Vargas-Mendoza, J. E., Mayoral-Garcia, M., MatadamasZarate, C., Elizarraras-Cruz, A., Taylor, M., et al. (2010). Psychological response of family members of patients hospitalised for influenza A/H1N1 in Oaxaca, Mexico. BMC Psychiatry 10:104. doi: 10.1186/1471-244X-10-104

Fernandez-Rouco, N., Carcedo, R. J., Lopez, F., and Orgaz, M. B. (2019). Mental health and proximal stressors in transgender men and women. J. Clin. Med. 8:413. doi: $10.3390 / \mathrm{jcm} 8030413$

Gariepy, G., Honkaniemi, H., and Quesnel-Vallee, A. (2016). Social support and protection from depression: systematic review of current findings in Western countries. Br. J. Psychiatry 209, 284-293. doi: 10.1192/bjp.bp.115.169094

Gater, R., Tansella, M., Korten, A., Tiemens, B. G., Mavreas, V. G., and Olatawura, M. O. (1998). Sex differences in the prevalence and detection of depressive and anxiety disorders in general health care settings: report from the World Health Organization Collaborative Study on Psychological Problems in General Health Care. Arch. Gen. Psychiatry 55, 405-413. doi: 10.1001/archpsyc.55.5.405

Gostic, K., Gomez, A. C., Mummah, R. O., Kucharski, A. J., and Lloyd-Smith, J. O. (2020). Estimated effectiveness of symptom and risk screening to prevent the spread of COVID-19. eLife 9:e55570. doi: 10.7554/eLife.55570

Hamilton, T. K., and Schweitzer, R. D. (2000). The cost of being perfect: perfectionism and suicide ideation in university students. Aust. N. Z. J. Psychiatry 34, 829-835. doi: 10.1080/j.1440-1614.2000.00801.x

Hirsch, J. K., and Barton, A. L. (2011). Positive social support, negative social exchanges, and suicidal behavior in college students. J. Am. Coll. Health 59, 393-398. doi: 10.1080/07448481.2010.515635

Huang, Y. Q., Dang, W. M., and Liu, Z. R. (2003). Psychosocial aspects in three universities during sars epidemic in beijing. Chinese Ment. Health J. 17, 521-523.

Jenkins, P. E., Ducker, I., Gooding, R., James, M., and Rutter-Eley, E. (2020). Anxiety and depression in a sample of UK college students: a study of prevalence, comorbidity, and quality of life. J. Am. Coll. Health [Epub ahead of print]. doi: 10.1080/07448481.2019.1709474

Kanadiya, M. K., and Sallar, A. M. (2011). Preventive behaviors, beliefs, and anxieties in relation to the swine flu outbreak among college students aged 18-24 years. Z. Gesundh. Wiss. 19, 139-145. doi: 10.1007/s10389-010-0373-3

Kawohl, W., and Nordt, C. (2020). COVID-19, unemployment, and suicide. Lancet Psychiatry 7, 389-390. doi: 10.1016/s2215-0366(20)30141-3

Kessler, R., Price, R., and Wortman, C. (1985). Social factors in psychopathology: stress, social support, and coping processes. Annu. Rev. Psychol. 36, 531-572. doi: 10.1146/annurev.ps.36.020185.002531

Khan, A., Farooqui, A., Guan, Y., and Kelvin, D. J. (2015). Lessons to learn from MERS-CoV outbreak in South Korea. J. Infect. Dev. Countr. 9, 543-546. doi: $10.3855 /$ jidc. 7278

Kim, S., Sinn, D., and Syn, S. Y. (2018). Analysis of college students' personal health information activities: online survey. J. Med. Internet Res. 20:e132. doi: 10.2196/jmir.9391

Lllhm, L. (2006). A Survey on University Students' Suicidal Ideation and the Influence Factors. Kirkland, WA: Northwest University.

Lai, J., Ma, S., Wang, Y., Cai, Z., Hu, J., Wei, N., et al. (2020). Factors Associated With Mental Health Outcomes Among Health Care Workers Exposed to Coronavirus Disease 2019. JAMA Netw. Open 3:e203976. doi: 10. 1001/jamanetworkopen.2020.3976

Lei, X. Y., Xiao, L. M., Liu, Y. N., and Li, Y. M. (2016). Prevalence of depression among chinese university students: a meta-analysis. PLoS One 11:e0153454. doi: 10.1371/journal.pone. 0153454

Leung, G. M., Lam, T. H., Ho, L. M., Ho, S. Y., Chan, B. H., Wong, I. O., et al. (2003). The impact of community psychological responses on outbreak control for severe acute respiratory syndrome in Hong Kong. J. Epidemiol. Community Health 57, 857-863. doi: 10.1136/jech.57.11.857
Lew, B., Osman, A., Huen, J. M. Y., Siau, C. S., Talib, M. A., Cunxian, J., et al. (2020). A comparison between American and Chinese college students on suicide-related behavior parameters. Int. J. Clin. Health Psychol. 20, 108-117. doi: 10.1016/j.ijchp.2020.03.005

Li, D. J., Ko, N. Y., Chen, Y. L., Wang, P. W., Chang, Y. P., Yen, C. F., et al. (2020). COVID-19-related factors associated with sleep disturbance and suicidal thoughts among the taiwanese public: a facebook survey. Int. J. Environ. Res. Public Health 17:4479. doi: 10.3390/ijerph17124479

Li, J., Lin, J., and Wang, X. (2003). Effects of catastrophic flood on mental health of students whose families were in disaster-afflicted areas. Chinese J. Sch. Health $24,456-457$.

Li, X., Shi, J. L., Xiong, W., Mu, Y. G., and Wei, X. (2011). Study on mental status for the nursing freshmen in a medical college after wenchuan earthquake. China J. Health Psychol. 19, 233-235.

Liao, Q., Cowling, B. J., Lam, W. W., Ng, D. M., and Fielding, R. (2014). Anxiety, worry and cognitive risk estimate in relation to protective behaviors during the 2009 influenza A/H1N1 pandemic in Hong Kong: ten cross-sectional surveys. BMC Infect. Dis. 14:169. doi: 10.1186/1471-2334-14-169

Liu, J. J., Bao, Y., Huang, X., Shi, J., and Lu, L. (2020). Mental health considerations for children quarantined because of COVID-19. Lancet Child Adolesc. Health 4, 347-349. doi: 10.1016/S2352-4642(20)30096-1

Main, A., Zhou, Q., Ma, Y., Luecken, L. J., and Liu, X. (2011). Relations of SARS-related stressors and coping to Chinese college students' psychological adjustment during the 2003 Beijing SARS epidemic. J. Couns. Psychol. 58, 410-423. doi: $10.1037 / \mathrm{a} 0023632$

Oboho, I. K., Tomczyk, S. M., Al-Asmari, A. M., Banjar, A. A., Al-Mugti, H., Aloraini, M. S., et al. (2015). 2014 MERS-CoV outbreak in Jeddah-a link to health care facilities. N. Engl. J. Med. 372, 846-854. doi: 10.1056/ NEJMoa1408636

Othman, N., Ahmad, F., El Morr, C., and Ritvo, P. (2019). Perceived impact of contextual determinants on depression, anxiety and stress: a survey with university students. Int. J. Ment. Health Syst. 13:17. doi: 10.1186/s13033-0190275-x

Parker, M., Duran, B., Rhew, I., Magarati, M., Larimer, M., and Donovan, D. (2021). Risk and Protective Factors Associated with Moderate and Acute Suicidal Ideation among a National Sample of Tribal College and University Students 2015-2016. J. Rural Health 37, 545-553. doi: 10.1111/jrh.12510

Pruitt, L. D., McIntosh, L. S., and Reger, G. (2020). Suicide safety planning during a pandemic: the implications of COVID-19 on coping with a crisis. Suicide Life Threat. Behav. 50, 741-749. doi: 10.1111/sltb.12641

Purtle, J. (2020). COVID-19 and mental health equity in the United States. Soc. Psychiatry Psychiatr. Epidemiol. 55, 969-971. doi: 10.1007/s00127-02001896-8

Recabarren, R. E., Gaillard, C., Guillod, M., and Martin-Soelch, C. (2019). Shortterm effects of a multidimensional stress prevention program on quality of life, well-being and psychological resources. A randomized controlled trial. Front. Psychiatry 10:88. doi: 10.3389/fpsyt.2019.00088

Ren, S. Y., Gao, R. D., and Chen, Y. L. (2020). Fear can be more harmful than the severe acute respiratory syndrome coronavirus 2 in controlling the corona virus disease 2019 epidemic. World J. Clin. Cases 8, 652-657. doi: 10.12998/wjcc.v8. i 4.652

Scanlan, J. N., and Hazelton, T. (2019). Relationships between job satisfaction, burnout, professional identity and meaningfulness of work activities for occupational therapists working in mental health. Aust. Occup. Ther. J. 66, 581-590. doi: 10.1111/1440-1630.12596

Shuiyuan, X. (1987). The influence of social support on physical and mental health. Chinese Ment. Health J. 1, 183-187.

Silverman, M. M., Meyer, P. M., Sloane, F., Raffel, M., and Pratt, D. M. (1997). The Big Ten Student Suicide Study: a 10-year study of suicides on midwestern university campuses. Suicide Life Threat. Behav. 27, 285-303.

Spitzer, R. L., Kroenke, K., and Williams, J. B. (1999). Validation and utility of a self-report version of PRIME-MD: the PHQ primary care study. Primary Care Evaluation of Mental Disorders. Patient Health Questionnaire. JAMA 282, 1737-1744. doi: 10.1001/jama.282.18.1737

Spitzer, R. L., Kroenke, K., Williams, J. B., and Lowe, B. (2006). A brief measure for assessing generalized anxiety disorder: the GAD-7. Arch. Intern. Med. 166, 1092-1097. doi: 10.1001/archinte.166.10.1092 
van Harmelen, A. L., Gibson, J. L., St Clair, M. C., Owens, M., Brodbeck, J., Dunn, V., et al. (2016). Friendships and family support reduce subsequent depressive symptoms in at-risk adolescents. PLoS One 11:e0153715. doi: 10.1371/journal. pone. 0153715

Wang, C., Pan, R., Wan, X., Tan, Y., Xu, L., Ho, C. S., et al. (2020). Immediate psychological responses and associated factors during the initial stage of the 2019 Coronavirus Disease (COVID-19) epidemic among the general population in China. Int. J. Environ. Res. Public Health 17:1729. doi: 10.3390/ ijerph17051729

Wang, Y., Zhao, X., Feng, Q., Liu, L., Yao, Y., and Shi, J. (2020). Psychological assistance during the coronavirus disease 2019 outbreak in China. J. Health Psychol. 25, 733-737. doi: 10.1177/1359105320919177

Xiang, Y. T., Yang, Y., Li, W., Zhang, L., Zhang, Q., Cheung, T., et al. (2020). Timely mental health care for the 2019 novel coronavirus outbreak is urgently needed. Lancet Psychiatry 7, 228-229. doi: 10.1016/S2215-0366(20)30046-8

Yang, Y., Li, W., Zhang, Q., Zhang, L., Cheung, T., and Xiang, Y. T. (2020). Mental health services for older adults in China during the COVID-19 outbreak. Lancet Psychiatry 7:e19. doi: 10.1016/S2215-0366(20)30079-1

Zhong, B. L., Luo, W., Li, H. M., Zhang, Q. Q., Liu, X. G., Li, W. T., et al. (2020). Knowledge, attitudes, and practices towards COVID-19 among Chinese residents during the rapid rise period of the COVID-19 outbreak: a quick online cross-sectional survey. Int. J. Biol. Sci. 16, 1745-1752. doi: 10.7150/ijbs.45221
Zhou, S. J., Zhang, L. G., Wang, L. L., Guo, Z. C., Wang, J. Q., Chen, J. C., et al. (2020). Prevalence and socio-demographic correlates of psychological health problems in Chinese adolescents during the outbreak of COVID-19. Eur. Child Adolesc. Psychiatry 29, 749-758. doi: 10.1007/s00787-020-01541-4

Conflict of Interest: The authors declare that the research was conducted in the absence of any commercial or financial relationships that could be construed as a potential conflict of interest.

Publisher's Note: All claims expressed in this article are solely those of the authors and do not necessarily represent those of their affiliated organizations, or those of the publisher, the editors and the reviewers. Any product that may be evaluated in this article, or claim that may be made by its manufacturer, is not guaranteed or endorsed by the publisher.

Copyright (c) 2021 Zhou, Wang, Qi, Yang, Gao, Zhang, Zhang, Yang and Chen. This is an open-access article distributed under the terms of the Creative Commons Attribution License (CC BY). The use, distribution or reproduction in other forums is permitted, provided the original author(s) and the copyright owner(s) are credited and that the original publication in this journal is cited, in accordance with accepted academic practice. No use, distribution or reproduction is permitted which does not comply with these terms. 